\title{
Optimum INR intensity and therapeutic INR control in patients with mechanical heart valve prosthesis on warfarin oral anticoagulation at Dr George Mukhari academic hospital: a three-year retrospective study
}

\author{
S Ntlokotsi ${ }^{a}$, MF Moshesh ${ }^{a}$, P Mntla $^{a}$, OA Towobola ${ }^{a *}$ and MA Mogale ${ }^{b}$ \\ ${ }^{a}$ Department of Internal Medicine, School of Medicine, Sefako Makgatho Health Sciences University, Pretoria, South Africa \\ ${ }^{b}$ Department of Biochemistry, School of Science \& Technology, Sefako Makgatho Health Sciences University, \\ Pretoria, South Africa \\ *Corresponding author, email: Olakunle.towobola@smu.ac.za
}

Background: Available evidence suggest that the optimum prothrombin time-international normalised ratio (PT-INR) intensities recommended for anticoagulation of patients with mechanical heart valve prosthesis may not apply to all race groups. Optimal PT-INR target ranges and effectiveness of warfarin oral anticoagulation were determined among black South African patients fitted with St Jude bileaflet mechanical heart valve prosthesis (SJBMHVP) at Dr George Mukhari Academic Hospital (DGMAH).

Methods: A convenience sample of 95 medical records of patients fitted with SJBMHVP from 1994 until 2013 was reviewed. Optimum PT-INR target ranges were estimated using two different methods: the classical two PT-INR target level method and the alternative, PT-INR specific incident rate method. The quality of warfarin anticoagulation was assessed using the fraction in therapeutic range method.

Results: Optimum PT-INR target ranges for all participants fitted with SJBMHVP in the aortic position was estimated to be 2.03.5 and 2.6-3.5, respectively, by the classical and alternative methods. That of the patients with mitral valve replacement was estimated to be in the range $2.6-3.5$ by the classical method and that of patients with double heart valve replacement was estimated to be $<3.5$ by both methods. The quality of warfarin anticoagulation of participants with SJBMHVP replacement was found to be inadequate as indicated by percentage time in treatment range (TTR) of $49.7 \%$ for all study participants compared with the ideal TTR of $70 \%$ and above.

Conclusion: Optimum Caucasian-based PT-INR intensities recommended for oral anticoagulation of patients fitted with mechanical heart valve prosthesis are applicable to black patients fitted with SJBMHVP at DGMAH.

Keywords: black South African patients, heart valve prosthesis, optimal PT-INR, time in treatment range, warfarin oral anticoagulation

\begin{abstract}
Introduction
In clinical practice, warfarin oral anticoagulation of patients with mechanical valve replacement is monitored through frequent laboratory measurement of prothrombin time and calculation of the prothrombin time-international normalized ratio (PTINR). ${ }^{1,2}$ The PT-INR values are then compared with the recommended optimum INR target ranges available in most treatment guidelines ${ }^{2-4}$ to allow for the timeous adjustment of warfarin dosage. Studies have established that the risk of bleeding increases with higher PT-INR values while the risk of thromboembolism and clotted prosthetic valves increases with lower PT-INR values. ${ }^{2,3}$ The risk of both thromboembolism and bleeding has also been shown to depend on the percentage of PT-INR in the treatment range-the so-called time in treatment range (TTR). ${ }^{1}$
\end{abstract}

Optimum PT-INR target ranges used in most developing countries for warfarin anticoagulation of patients with mechanical heart valve replacement are those recommended by either the American Heart Association/American College of Cardiology $(\mathrm{AHA} / \mathrm{ACC})^{3}$ or the European therapeutic guidelines. ${ }^{4-6}$ These PT-INR intensities are based on randomised clinical trials conducted among Caucasian subjects in developed countries $3,5,7$ Several studies conducted among Asian populations have, however, suggested that optimal PT-INR for warfarin oral anticoagulation of patients with mechanical heart valve replacement may be race dependent. ${ }^{8-11}$ This further suggest that the optimum PT-INR intensities recommended by both the AHA/ $\mathrm{ACC}^{3}$ and European guidelines ${ }^{4,5}$ may not necessary apply to black South African patients with mechanical heart valve prosthesis. To the best knowledge of the authors of this article, no study has ever been conducted to determine or estimate the optimum PT-INR target ranges for warfarin oral anticoagulation of mechanical heart valve prosthesis in a public health setting that caters for mostly black African people, such as Dr George Mukhari Academic Hospital (DGMAH). Thus, the objectives of the study were to determine the three-year (January 2012January 2015) incident rates of both thromboembolic and haemorrhagic events, to estimate the PT-INR target ranges for warfarin oral anticoagulation and to determine effectiveness of warfarin oral anticoagulation among black South African patients fitted with SJBMHVP at DGMAH.

\section{Methodology}

\section{Study design and sample}

A retrospective, research design was adopted for the current study. In brief, a convenient sample of 95 medical records of patients who were fitted with SJBMHVP from 1994 to 2013, and were still being followed up for warfarin oral anticoagulation 
monitoring and prevention of mechanical heart valve related complications during the three-year retrospective study period (from January 1, 2012 to December 31, 2014), were reviewed. The study sample was divided into three groups: a group consisting of patients with St Jude bileaflet mechanical (SJbm) aortic valve replacement (AVR group), a group consisting of patients with SJbm mitral valve replacement (MRV group) and a group consisting of patients with SJbm aortic and SJbm mitral valve replacement (double valve replacement (DVR group). The warfarin used during the period of this study was supplied solely by Roche Pharmaceutical, South Africa (Couma$\left.\operatorname{din}^{\oplus}\right)$. Compliance by patients for accurate use of the prescribed warfarin therapy was assessed using the following procedures: (a) patients were reviewed on a monthly basis; (b) INR levels were determined at each monthly visit; (c) where possible patients were instructed to bring empty packets of the drug; (d) any evidence of bleeding from the gum or skin bruises was noted.

\section{Data collection}

Relevant demographic and clinical data (type and position of mechanical heart valve prosthesis, date of heart valve replacement, duration of follow-up period, warfarin dosage, PT-INR values and nature as well as the date of occurrence of anticoagulation-related complications) were recorded. The recorded information was used for calculation of incident rates of warfarin anticoagulation-related thromboembolic and haemorrhage events, estimation of optimum PT-INR target ranges and determination of TTR. Incident rates of both valve-related thromboembolic and haemorrhagic events among the study participants were calculated by dividing the total number of events during the study period by patient years. Patient years were in turn determined by multiplying the sum of follow-up time for all patients (from the time of valve replacement surgery or since the occurrence of a previous valve-related complication until the occurrence of a new or recurrent valve-related complication or the end of the study period) by the total number of patients.

Optimum PT-INR target ranges for warfarin anticoagulation of patients fitted with an SJbm mechanical heart valve replacement were estimated using two different methods. In the first method described by both Altman et al. ${ }^{12}$ and Turpie et al., ${ }^{13}$ averages of PT-INR values before and immediately after the occurrence of a valve-related complication were taken as the anticoagulation related PT-INR values at which the said complication had occurred. These average PT-INR values were categorised into three categories: a category with PT-INR values < 2.0; a category with PT-INR values between 2.0 and 3.5 and a category with PT-INR values $>3.5$ as recommended by AHA/ACC guidelines. ${ }^{3}$ The percentages of PT-INR in each category were then determined and presented in the form of a pie chart from which the optimal PT-INR target ranges for prevention of both thromboembolic and haemorrhagic valve-related complications were estimated. In the second method, PT-INRspecific incident rates of all valve-related complications (all thromboembolic events and all haemorrhagic events) were determined by the method described by Rosendaal et al. ${ }^{14}$ In brief, PT-INR values at which valve-related complications had occurred were categorised into the following ranges: $(<2.0)$; (2.0-2.5); (2.6-3.0); (3.1-3.5); (3.5-4.0); (4.1-4.5); (4.5-5.0); (5.0$6.0)$ and $(>6.0)$. The frequency of valve-related complications in each of the above-mentioned PT-INR categories was determined and plotted against PT-INR categories. From the resultant plot, the PT-INR range(s) with the lowest frequency of valverelated complications was regarded as the optimum PT-INR range for the study participants. The quality of warfarin anticoagulation of the study participants was assessed according to the time in treatment range method described by Schimitt et al. ${ }^{1}$ In brief, the fraction of PT-INR values within range was calculated by dividing the number of PT-INR values within the optimum target recommended range of 2.0-3.5 for all the study groups by the total number of PT-INR values during the three-year study period and multiplying this by 100 .

\section{Data analysis}

Categorical variables were expressed as counts (frequencies) and percentages. Continuous variables were expressed as mean \pm standard deviation (SD) and incident rates were expressed as percentages per patient years. Percentages were compared using the chi-square test and means were compared using the analysis of variance (ANOVA). $P$-values of $<0.05$ were considered statistically significant. Data were analysed using the Statistical Package for Social Science (SPSS ${ }^{\oplus}$ ) version 24 for Windows (IBM Corp, Armonk, NY, USA).

\section{Results}

\section{Demographics and clinical characteristics of the study subjects}

Demographic and the relevant clinical parameters of the three study groups are summarised in Table 1 . The mean age of the

Table 1: Demographic and relevant clinical characteristics of study participants

\begin{tabular}{|c|c|c|c|c|c|}
\hline Factor & AVR group & MVR group & $\begin{array}{c}\text { DVR } \\
\text { group }\end{array}$ & $p$-value & $\begin{array}{c}\text { All } \\
\text { subjects }\end{array}$ \\
\hline Number: $n(\%)$ & $7(7.4)$ & $53(55.8)$ & $35(36.8)$ & 0.562 & 95 \\
\hline Age at surgery (mean $\pm S D$ ) years & $43.9 \pm 16.1$ & $37.1 \pm 13.6$ & $38.0 \pm 13.2$ & 0.294 & $39.7 \pm 18.6$ \\
\hline \multicolumn{6}{|l|}{ Gender: } \\
\hline Female: $n(\%)$ & $2(28.6)$ & $44(83.0)$ & $21(60.0)$ & 0.321 & $67(70.5)$ \\
\hline Males: $n(\%)$ & $5(71.4)$ & $9(17.0)$ & $14(40.0)$ & 0.241 & $28(29.5)$ \\
\hline Oral Warfarin dosage (mg/day) (mean \pm SD) & $3.93 \pm 1.33$ & $4.33 \pm 1.21$ & $4.07 \pm 1.22$ & 0.498 & $4.14 \pm 1.25$ \\
\hline PT-INR (Mean \pm SD) & $2.29 \pm 0.62$ & $2.42 \pm 0.50$ & $2.54 \pm 0.60$ & 0.476 & $2.42 \pm 0.74$ \\
\hline Alcohol intake: $n(\%)$ & $2(28.6)$ & $5(9.43)$ & $3(8.57)$ & 0.210 & $10(10.5)$ \\
\hline Smoking: $n$ (\%) & $1(14.3)$ & $6(11.3)$ & $5(14.3)$ & 0.198 & $12(12.6)$ \\
\hline Patients followed up for > 10 years since valve replacement surgery: $n(\%)$ & $3(42.8)$ & $34(66.0)$ & $15(42.8)$ & 0.634 & $52(54.7)$ \\
\hline Patients followed-up for 5-10 years since valve replacement surgery: $n(\%)$ & $3(42.8)$ & $24(45.3)$ & $9(25.7)$ & 0.138 & $36(37.9)$ \\
\hline Patients followed up for $<5$ years since valve replacement surgery: $n(\%)$ & $1(14.3)$ & $6(11.3)$ & $10(28.6)$ & 0.282 & $17(17.9)$ \\
\hline
\end{tabular}


study participants at time of valve replacement surgery was $39.7 \pm$ 18.5 years. Of the 95 patients whose medical records were reviewed, 7 underwent a single aortic-valve replacement (AVR group), 53 underwent single mitral-valve replacement (MVR group) and 35 underwent double valve replacement (DVR group III) with SJbm heart valve devices.

At the time of the study, 52 patients ( 3 with AVR, 34 with MVR and 15 with DVR) had been followed up for more than 10 years since valve replacement surgery, 36 patients ( 3 with AVR, 24 with MVR and 9 with DVR) had been followed up for between 5 and 10 years since valve replacement surgery and 17 patients ( 1 with AVR, 6 with MVR and 10 with DVR) had been followed for a period of less than 5 years since valve replacement surgery. The mean PT-INR values for the AVR, MVR and DVR groups during the three-year study period were respectively, $2.29 \pm 0.62,2.42 \pm 0.5$ and $2.54 \pm 0.6$ with no significant difference among the three groups $(p>0.05)$. The corresponding mean $\pm S D$ oral warfarin dosages for the three groups were $3.93 \pm 1.3,4.33 \pm 1.21$ and $4.07 \pm 1.22$ per day with no significant differences among the groups ( $p>0.05$; ANOVA). Only a small number of the total study subjects had either a history of alcohol intake or cigarette smoking $(10.5 \%$ and $12.6 \%$ respectively) (see Table 1).

Clinical co-morbidities, which are also risk factors for valverelated complications recorded in patients' medical records, are tabulated in Table 2. It can be seen from Table 2 that the most common clinical co-morbidities recorded for all study subjects were hypertension (41.1\%) followed by atrial fibrillation (18.9\%) and history of previous stroke (7.36\%).

\section{Incident rates of warfarin anticoagulation-related complications}

Haemorrhagic complications occurred in all the study groups whereas thromboembolic complications did not occur in the DVR group (Figure 1). The incident rates of both thromboembolic and haemorrhagic events were significantly higher in the AVR group compared with the other study groups $(p<0.05)$. In patients with AVR and MVR the most common thromboembolic complication was ischaemic cerebral stroke while the most common haemorrhagic complication in these groups was haemorrhagic cerebral stroke. The incident rate of haemorrhagic complications was significantly higher in the AVR group than in the other study groups $(p<0.05)$. The most common haemorrhagic complication in patients with DVR was gastrointestinal bleeding. With regard to all study participants with mechanical heart valve replacement the most common thromboembolic complication observed was ischaemic cerebral stroke and the most commonly observed haemorrhagic complications were haemorrhagic stroke and gastrointestinal bleeding. Furthermore, the incident rates of both thromboembolic and

Table 2: Clinical co-morbidities recorded in medical records of the study subjects

\begin{tabular}{|c|c|c|c|c|}
\hline $\begin{array}{l}\text { Clinical co- } \\
\text { morbidities }\end{array}$ & $\begin{array}{c}\text { Group } \\
\text { I } \\
\text { (AVR) } \\
n(\%)\end{array}$ & $\begin{array}{c}\text { Group II } \\
\text { (MVR) } \\
n(\%)\end{array}$ & $\begin{array}{c}\text { Group } \\
\text { III } \\
\text { (DVR) } \\
n(\%)\end{array}$ & $\begin{array}{c}\text { All } \\
\text { subjects } \\
n(\%)\end{array}$ \\
\hline Atrial fibrillation & $0(0.0)$ & $12(22.6)$ & $6(17.1)$ & $18(18.9)$ \\
\hline Previous stroke & $1(14.3)$ & $5(9.43)$ & $1(2.86)$ & $7(7.36)$ \\
\hline Hypertension & $5(71.4)$ & $20(37.7)$ & $14(14.0)$ & $39(41.1)$ \\
\hline Diabetes & $0(0.0)$ & $0(0.0)$ & $1(2.86)$ & $1(1.05)$ \\
\hline
\end{tabular}

haemorrhagic complications appeared to be negatively associated with the number of patients followed up in each study group, i.e. the fewer the number of patients followed up in each study group, the higher the incident rate in the group. None of the patients with DVR had a thromboembolic event. None of the observed co-morbidities was associated with increased risk of thromboembolic events in the current study.

\section{Optimal PT-INR ranges for the study subjects}

Optimal PT-INR range for the study participants was estimated using two different methods as described in the methodology section. The results of the first method ${ }^{8,9,12}$ are summarised in Figure 2 (thromboembolic and haemorrhagic events). From Figure 2 it can be seen that most of the thromboembolic complications (75\%) observed during the study period occurred at PT-INR values of less than 2.0, and most of the haemorrhagic complications (69.2\%) occurred at PT-INR values $>3.5$. Thus, on the basis of the results presented here, the PT-INR range of 2.0 to 3.5 can be regarded as the optimal PT-INR for the study participants.

For the second method, ${ }^{14,15}$ PT-INR-specific frequencies of all valve-related complications that were recorded during the study period are tabulated in Table 3.

A plot of the PT-INR-specific frequency of PT-INR at which all valve-related complications occurred in the MVR group, DVR group and all study subjects is shown in Figure 3.

Both Table 3 and Figure 3 show that for all study participants with mechanical heart valve replacement the lowest frequency

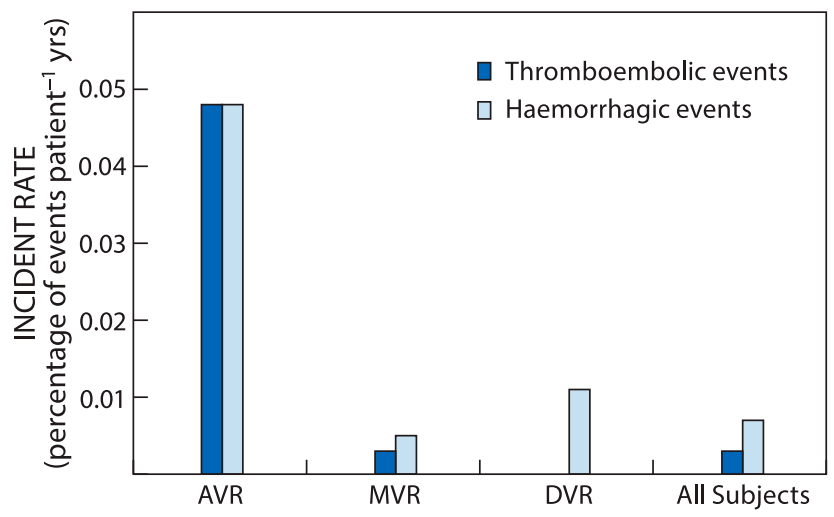

Figure 1: Incident rates of the total thromboembolic and haemorrhagic complications among the different study groups.

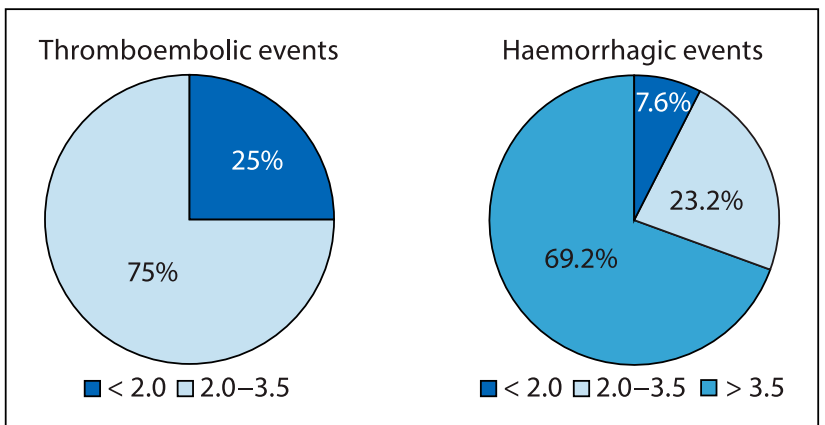

Figure 2: The occurrence of thromboembolic and haemorrhagic events 
Table 3: PT-INR-specific frequency of all valve-related complications

\begin{tabular}{lc}
\hline $\begin{array}{l}\text { PT- INR } \\
\text { range }\end{array}$ & $\begin{array}{c}\text { Frequency of all valve-related complications in each } \\
\text { PT-INR range }\end{array}$ \\
\hline$<2.0$ & 4 \\
\hline $2.1-2.5$ & 1 \\
$2.6-3.0$ & 1 \\
\hline $3.1-3.5$ & 0 \\
\hline $3.5-4.0$ & 2 \\
\hline $4.1-4.5$ & 2 \\
\hline $4.5-5.0$ & 1 \\
\hline $5.0-6.0$ & 2 \\
$>6.0$ & 5 \\
\hline
\end{tabular}

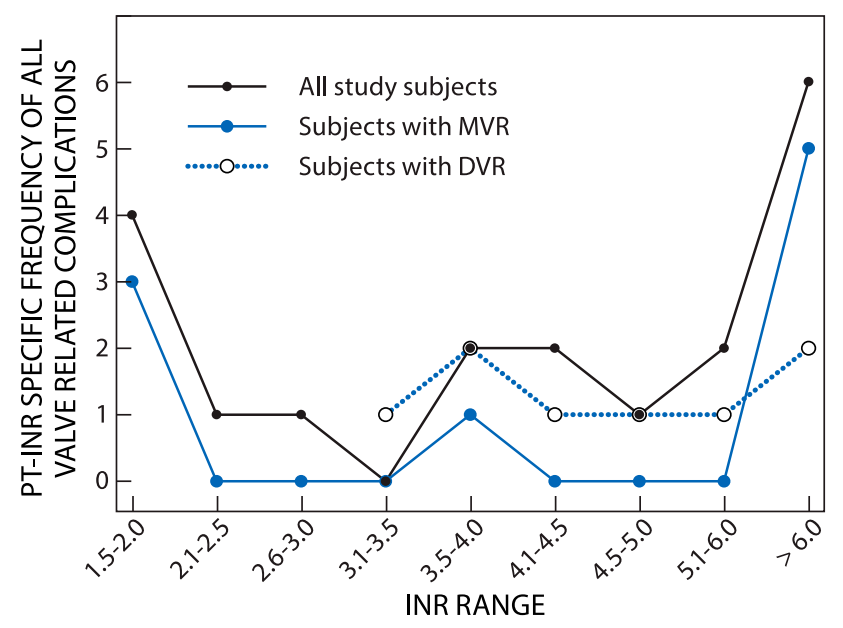

Figure 3: Optimum PT-INR range by the alternative method (only two study subjects with AVR had valve-related complications, hence a meaningful curve could not be constructed from the corresponding two points).

of all valve-related complications occurred at the PT-INR range 2.6-3.5. For the DVR group which had only haemorrhagic events, no event was observed for the INR values below 3.5. For the MVR group no event was observed in the PT-INR ranges 2.1-3.5 and 4.1-6.0. Thus, on the basis of this second method of estimating the optimal PT-INR range, the range 2.6-3.5 was regarded as the optimal PT-INR for all the patients with mechanical heart valve replacement at this institution (DGMAH).

\section{Quality of warfarin anticoagulation}

In order to assess the quality of warfarin anticoagulation of patients with mechanical heart valve prosthesis at DGMAH during the three-year retrospective study period, percentages of PT-INR values within the AHA $/ \mathrm{ACC}^{5}$ recommended target range of 2.0-3.5, percentage of PT-INR values below the lower limit of the recommended target range and percentage of PTINR values above the upper limit of the recommended target range were calculated for the different study groups and the results are summarised in Table 4. As shown in Table 4, the time in treatment range for the patients with AVR, MVR, DVR and all study subjects were $22.6 \%, 53.1 \%, 50.8 \%$ and $49.7 \%$ respectively. All these percentages are significantly lower than $70 \%$, the recommended limit of adequate or good quality of anticoagulation. It can also be seen in Table 4 that for all the study groups the percentage of PT-INR below the target range is higher than the percentages of PT-INR above the target range.
Table 4: Assessment of quality of anticoagulation of the study subjects with mechanical heart valve prosthesis

\begin{tabular}{llllcc}
\hline Factor & AVR & MVR & DVR & $\begin{array}{c}p \text { - } \\
\text { value }\end{array}$ & $\begin{array}{c}\text { All } \\
\text { subjects }\end{array}$ \\
\hline $\begin{array}{l}\text { Total number of } \\
\text { PT-INRs during the } \\
\text { study period }(n)\end{array}$ & 93 & 604 & 419 & & 1116 \\
$\begin{array}{l}\text { Number of PT-INRs } \\
\text { within the target } \\
\text { range of 2.0-3.5 }(n)\end{array}$ & 21 & 321 & 213 & & 555 \\
$\begin{array}{l}\text { Number of PT-INRs } \\
\text { below PT-INR } \\
\text { target range }\end{array}$ & 42 & 203 & 154 & & 399 \\
$\begin{array}{l}\text { Number of PT-INRs } \\
\text { above target ( } n \text { ) }\end{array}$ & 30 & 80 & 52 & & 162 \\
$\begin{array}{l}\text { Time in treatment } \\
\text { range (\%) }\end{array}$ & 22.6 & 53.1 & 50.8 & 0.867 & 49.7 \\
$\begin{array}{l}\text { Time below } \\
\text { treatment range } \\
\text { (\%) }\end{array}$ & 45.2 & 33.6 & 36.8 & 0.378 & 35.7 \\
$\begin{array}{l}\text { Time above } \\
\text { treatment range } \\
\text { (\%) }\end{array}$ & 32.3 & 13.2 & 12.4 & 0.413 & 14.5 \\
\hline
\end{tabular}

\section{Discussion}

In agreement with the results of previous similar studies, ${ }^{9,10,13}$ the most common thromboembolic event observed among all the study groups was ischaemic cerebral stroke. Previous studies found and reported that the incident rate of thromboembolic events is highest for patients with DVR, followed by that in patients with MVR and then that in patients with AVR. ${ }^{2,15}$ In contrast with these findings, the current study observed the highest incidence of ischaemic stroke in patients with AVR $(0.049 \%$ per patient year), followed by those with MVR (0.002\% per patient year). No thromboembolic event was observed in patients with DVR. This discrepancy could be attributed to a number of factors including the length of follow-up after valve replacement surgery and the small number of patients in each of our study groups. In this regard the followup time in patient years was much lower for the AVR (291 cumulative patient years) group in comparison with the MVR (24 589 cumulative patient years) and DVR (1 624 cumulative patient years) groups.

The most common thromboembolic event was cerebral ischaemic stroke and the most common bleeding complications were haemorrhagic stroke and gastrointestinal bleeding. Gastrointestinal bleeding was particularly more common in patients with DVR. This observation was unexpected since none of the study subjects were taking antiplatelet medications. The observed incidence of bleeding events associated with warfarin anticoagulation in the current study was higher in the AVR group (0.049\% per patient year), followed by that in the DVR group $(0.011 \%$ per patient year) and lowest in the MVR group ( $0.005 \%$ per patient year). This observation could be due to the same reasons which accounted for the differences in the incidence of thromboembolic events among the different study groups in the current study, i.e. the differences in the number of study subjects and follow-up time since valve replacement surgery.

The incidences of both thromboembolic and haemorrhagic complications observed among all study participants $(0.002 \%$ and $0.006 \%$ per patient-year respectively) were much lower than those reported in other similar previous studies. ${ }^{8-11,13}$ 
This discrepancy could be attributed to factors such as the length of follow-up since valve-replacement surgery and the fact that only patients fitted with the SJbm valve devices were enrolled in the current study. Some of the previous studies either did not specify the type of mechanical heart device used or used devices other than the St Jude device. ${ }^{8,9,11}$ Therefore, comparison of the findings of these studies with the findings of the current study would be misleading.

Optimum PT-INR intensities for warfarin anticoagulation of patients with mechanical heart valve replacement are reported to depend on several factors including the position of the heart valve. ${ }^{3,4}$ For the mechanical valves at different heart positions the optimal PT-INR intensities for Caucasian populations are 2.5 (range 2.0-3.0) for patients with AVR with no risk factors and 3.0 (range 2.5-3.5) for patients with AVR and risk factors and for patients with MVR and DVR with or without risk factors. The corresponding optimum PT-INR for Asian patients is reported to be much lower, at 1.6 (range $1.4-1.8)^{8-11}$ than those recommended for the Caucasian populations. No such range of optimal PT-INR intensity has previously been reported for patients of African descent. The authors could speculate on the basis of the values found in the current study that the range for African patients would fall between values quoted for Caucasians and those reported for Asian patients.

The quality of anticoagulation over time is usually assessed in terms of percentage time in therapeutic INR range (TTR). ${ }^{1,2}$ Patients with low-percentage TTR (a reflection of large variation in PT-INR over time) are at a higher risk of warfarin-related major bleeding and thromboembolic events. ${ }^{2}$ In the current study, the quality of warfarin anticoagulation of patients fitted with the St Jude bileaflet mechanical heart valve at DGMAH was found to be poor or inadequate with only $49.7 \%$ of all PT-INR results recorded during the three-year study period lying within the optimum PT-INR target range of 2.0-3.5. It was, however, observed in the current study that the tendency of physicians at DGMAH was towards under-coagulation rather than overcoagulation $35.7 \%$ time below therapeutic range versus $14.5 \%$ time above treatment range) of patients with mechanical heart valve replacement. The reason for the continuous undercoagulation by physicians at DGMAH observed in the current study could be the need to avoid bleeding events known to be associated with over-coagulation in clinical practice.

\section{Study limitations}

The findings from this study might have been influenced by several limitations. First, the sample size was small, thereby making it difficult to generalise the findings. Second, this was a retrospective study over a set time, thereby inevitably raising the concern of availability of all medical records. Third, some major valve-related complications could have been missed as a result of the exclusion of medical records of patients with missing data. Despite these limitations, the current study provides a valuable insight into the management of patient with mechanical heart valve replacement at DGMAH.

\section{Conclusion}

The optimum PT-INR target ranges estimated in the current study of between 2.0 and 3.5 overlap with those recommended by the Caucasian-based guidelines. ${ }^{2-4}$ This finding suggests that the optimum Caucasian based PT-INR intensities recommended for oral anticoagulation of patients fitted with mechanical heart valve prosthesis are applicable to black patients fitted with SJBMHVP at DGMAH.

Disclosure statement - No potential conflict of interest was reported by the authors.

Acknowledgement - The authors are grateful to the Department of Patient Administration of DGMAH for making available the medical records of patients that were used in the current study. The Department of Internal Medicine at Sefako Makgatho Health Sciences University is also acknowledged for granting $\mathrm{Dr}$ S. Ntlokotsi an opportunity to conduct an MMED Degree project from which the current study emanates.

\section{References}

1. Schimitt L, Speckman J, Ansel J. Quality assessment of anticoagulation dose management Comperative evaluation of measures of time in treatment range. Journal of thrombosis and thromboembolysis. 2003;15(3): 213-316. 10.1023/B:THRO.0000011377.78585.63

2. Wieloch $M$, Själander A, Frykman V, et al. Anticoagulation control in Sweden: reports of time in therapeutic range, major bleeding, and thrombo-embolic complications from the national quality registry AuriculA. Eur Heart J. 2011;32(18): 2282-2289. 10.1093/eurheartj/ ehr134

3. American Heart Association/American College of Cardiology. AHA/ ACC Guidelines for the Management of Patients with Valvular Heart Disease: Executive Summary. Circulation 2006;114: 450-527.

4. Butchart EG, Chohlke-Barvolff CG, Antunes MJ, et al Recommendations for the management of patients of patients after heart valve surgery. Eur Heart J. 2005;20: 263-271.

5. Butchart EG. Antithrombotic management in patients with prosthetic heart valves: A comparison of American and European guidelines. Heart. 2009:95: 430-436.

6. Roudaut R, Serr K, Lafitte S. Thrombosis of prosthetic heart valves: diagnosis and therapeutic considerations. Heart. 2007;93(1): 137142. $10.1136 /$ hrt.2005.071183

7. Mehta SR, Weitz Jl. Warfarin after bioprosthetic aortic valve implantation. Lancet. 2012; 308:2147-2148 10.1001/jama.2012.91476

8. Mori $\mathrm{T}$, Asano $\mathrm{M}$, Ohtake $\mathrm{OH}$, et al. Anticoagulant therapy after pros thetic heart valve replacement: Optimal PT-INR in Japanese patients. Ann Thorac Cardiovasc Surg. 2002;8: 83-87.

9. Tsai $\mathrm{Y}-\mathrm{T}$, Lee $\mathrm{C}-\mathrm{H}$, Hong G-J, et al. Long-term experience of anticoagulation therapy in Chinese patients with mechanical valves. J Med Sci. 2007;27(6): 259-264.

10. Akhtar RP, Abid PR, Zafar H, et al. Anticoagulation in patients following prosthetic heart valve replacement. Ann Thorac Cardiovasc Surg. 2009;15: 10-17.

11. Taksinachaneki S, Chaichun J, Hinwiset W, et al. Optimal PT-INR after prosthetic heart valve replacement: Queen Sirikit Heart Center's experience. The Thai Journal of Surgery. 2010;31: 54-57.

12. Altman R, Rouvier G, Gurfinkel E, et al. Comparison of two levels of anticoagulation therapy in patients with substitutes heart valves. J Thorac Cardiovasc Surg. 191;101: 427-431.

13. Turpie AGG, Gunstensen J, Hirsh J, et al. Randomized comparison of two intensities of oral anticoagulant therapy after tissue heart valve replacement. Lancet, 1988; 1:1242-1242. 10.1016/S0140-6736 (88)92070-3

14. Rosendaal FR, Cannegieter SC, van der Meer FJ, et al. A method to determine the optimal intensity of oral anticoagulant therapy. Thromb Haemost, 1993; 69(3):236-239.

15. Poller L. International normalized ratio (INR): the first 20 years. J Thromb Haemostat. 2004;2: 849-860. 10.1111/jth.2004.2.issue-6 